\title{
PERFORMANCE EVALUATION OF BANKS THROUGH FOUR PHASED DEA - A CASE STUDY
}

\author{
Hari Krishna Tata and Vijaya Sai Nimmagadda \\ Department of Mechanical Engineering, \\ V.R. Siddhartha College of Engineering, Vijayawada, India
}

\begin{abstract}
Evaluation and analysis of overall performance of banks is necessary to identify their healthy financial condition. In the present paper the overall performance of all the 26 Public sector banks in India is analyzed using four phase Data envelopment analysis (DEA). The necessary data for the period 2009-2014 is collected from IBA bulletin, statistics published by Reserve Bank of India and annual reports published by the banks. The evaluation factors are categorized as inputs and outputs to find the overall efficiency of the banks. The study made in this paper is used to improve the performance of the banks to come up to the desired standards.
\end{abstract}

Key words: Performance Evaluation; Data Envelopment Analysis, Closeness Coefficient; Attributes; Efficiency.

Cite this Article: Hari Krishna Tata and Vijaya Sai Nimmagadda. Performance Evaluation of Banks through Four Phased DEA - A Case Study, International Journal of Industrial Engineering Research and Development, 7(1), 2016, pp. 24-34.

https://iaeme.com/Home/issue/IJIERD?Volume $=7 \&$ Issue $=1$

\section{INTRODUCTION}

In India, Banking sector has been a significant driver of Gross Domestic Product (GDP) growth and it is an integral part of Indian financial system. Public Sector Banks are playing the pivotal role in the development by achieving multi societal and economic objective, as they promotes and develops the Small-Scale and Large-Scale industries, Entrepreneurs, Economic activities in backward regions and Enhances the foreign trade in India.

To measure the financial position of each bank and manage it efficiently and effectively so many efforts have been made from time to time. In the process of continuous evaluation of the bank's financial performance the academicians, scholars and administrators have made several studies. Marbini et al. (2010) evaluated a set of IT investment strategies using fuzzy DEA with TOPSIS feature and he concluded that DEA is an excellent methodology for measuring relative efficiencies of a set of 
DMUs due to its solid underlying theoretical basis and wide application to real world problems. Prasad and Ravinder (2012) analyzed a study entitled "A CAMEL Model Analysis of Nationalized Banks in India". The performance of twenty nationalized banks in India was analyzed through CAMEL model for the period from 2005-2010. Mohammed anwaral kabir (2012) analyzed the performance of financial and commercial banks in Bangladesh using CAMEL rating. Majid Kasimzadeh (2012) examined the efficiency of Indian Commercial banks during 2000-2010 by utilizing DEA and confirmed that selected Public Sector Banks are more efficient than Private Sector during the study period. Valeric Vierstraete (2012) computed Efficiency in Human Development as Human Development Index using Data Envelopment Analysis. Chen et al. (2013) estimated the management achievement of Taiwan banking under market risk using Fuzzy DEA. Iman Dabashi et al. (2013) evaluated the banks using DEA by taking fixed assets \& total deposits as input variables and total loans \& net income as output variables and analyzed their efficiencies in both CCR and BCC models. CA. Ruchi Gupta (2014) evaluated the performance of 26 Public sector banks by using CAMEL approach for a five year period from 2009-2013 and found that there is a statistically significant difference between the CAMEL ratios and thus the performance of all the Public financial institutions. Bhattacharya and Chakraborty (2014) evaluated the performance of eight IITs using a combined approach of DEA and TOPSIS, and he concluded that it can be applied in all the educational institutions and manufacturing organizations, as it has strong mathematical foundation. Ritika et al. (2014) presented an application of the output oriented constant returns to scale DEA model for measuring the organizational performance of twenty Indian microfinance Institutions.

Performance evaluation of Banks is a major concern for the managers, share holders, creditors, employees and customers, as strong banking system effects the growth and financial stability of the country. There is limited research, in the application of hybrid multi-criteria decision making methods for evaluation of performance of banks. Hence in this paper, TOPSIS method is integrated with DEA to evaluate performance of public sector banks.

\section{METHODOLOGY}

The methodology for evaluation of public sector banks is explained in the following steps.

Step 1: Determination of the best relative efficiency of Ideal bank $\left(\theta^{*}\right)$

Thus the mathematical model to find best relative efficiency of Ideal bank is

$\theta_{l}^{*}=\max \sum_{r=1}^{s} u_{r} y_{r}^{\max } \quad$ Subject to

$\sum_{i=1}^{m} v_{i} x_{i}^{\text {min }}=1 \quad \sum_{r=1}^{s} u_{r} y_{r p}-\sum_{i=1}^{m} v_{i} x_{i p} \leq 0, \forall j$,

$u_{r}, v_{i} \geq \varepsilon, \quad \forall \mathrm{r}, \mathrm{i}$ the $\mathrm{i}^{\text {th }}$

Where ur $(r=1, \ldots, s)$ and vi $(i=1, \ldots, m)$ are the weights of the $r^{\text {th }}$ output and Input respectively, and ' $\in$ ' is a small non Archimedean value, with each bank $(j=1$. $., \mathrm{n})$.

Step 2: Determination of the worst relative efficiency of nadir bank $\left(\varphi^{*}{ }^{*}\right)$ Thus the mathematical model to find worst relative efficiency of nadir bank is. 
$\varphi_{N}^{*}=\min \sum_{r=1}^{s} u_{r} * y_{r}{ }^{\min }$

s.t

$\sum_{i=1}^{m} v_{i}^{*} x_{i}^{\max }=1$

$\sum_{r=1}^{s} u_{r} * y_{r}^{\max }-\sum_{i=1}^{m} v_{i} * x_{i}^{\min } * \theta^{*}{ }_{l} \geq 0 \quad \forall j$

$\sum_{r=1}^{s} u_{r} * y_{r j}-\sum_{i=1}^{m} v_{i} * x_{i j} \leq 1 \quad \forall j$

$u_{r}, v_{i} \geq \in, \quad \forall r, i$

Step 3: Determination of the best relative efficiency of $i^{\text {th }}$ Bank $\left(\theta_{p}{ }_{p}\right)$

Thus the mathematical model to find best relative efficiency of $i^{\text {th }}$ bank is

$\theta_{p}^{*}=\max \sum_{r=1}^{s} u_{r} * y_{r p}$

S.t

$\sum_{i=1}^{m} v_{i} * x_{i p}=1$

$\sum_{r=1}^{s} u_{r} * y_{r}^{\max }-\sum_{i=1}^{m} v_{i} * x_{i}^{\min } * \theta_{l}^{*}=0$

$\sum_{r=1}^{s} u_{r} * y_{r j}-\sum_{i=1}^{m} v_{i} * x_{i j} \leq 1 \quad \forall p \quad u_{r}, v_{i} \geq \epsilon$

$\forall r, i$

Step 4: Determination of the worst relative efficiency of each bank $\left(\varphi_{p}^{*}\right)$

The mathematical model to determine the worst relative efficiency of each bank

$\varphi_{p}^{*}=\min \sum_{r=1}^{s} u_{r} * y_{r p}$

s.t

$\sum_{i=1}^{m} v_{i}^{*} x_{i p}=1$

$\sum_{r=1}^{s} u_{r} * y_{r}^{\min }-\sum_{i=1}^{m} v_{i} * x_{i}^{\max } * \varphi_{N}^{*}=0$

$\sum_{r=1}^{s} u_{r} * y_{r j}-\sum_{i=1}^{m} v_{i} * x_{i j} \leq 0 \quad \forall p$

$u_{r}, v_{i} \geq \in$

$\forall r, i$ 
Step 5: Calculation of the relative closeness of the banks using the mathematical models developed in step 1, step 2, step 3 and step 4 and are solved using LINGO solver. Then the relative closeness index of jth bank $(R C j)$ is calculated' using the following equation.

$$
R C_{j}=\frac{\left(\varphi_{j}^{*}-\varphi_{N}^{*}\right)}{\left(\varphi_{j}^{*}-\varphi_{N}^{*}\right)+\left(\theta_{l}^{*}-\theta_{j}^{*}\right)}
$$

\section{PERFORMANCE EVALUATION OF BANKS}

In the present paper the banks are evaluated using the criteria namely, Capital Adequacy, Asset Quality, Management Capability, Earnings Ability, and Liquidity. The sub-criteria under the above mentioned criteria are Capital Adequacy ratio, Advances to assets, Govt. security. to total Investment, Business per Employee, Profit per Employee, Credit Deposit Ratio, Return on Net worth, Return on Assets, NIM to Total Assets, Operating profit to total assets, Interest income to total income, Liquid Assets to Total Assets, Govt. sec. to total assets, Liquid Assets to total deposits, Liquid Assets to demand Deposits, Debt-Equity Ratio, Net NPA to Net Advances, Net NPA to Total Assets, and Total Investments to Total Assets. Secondary source of data collection from 2009-2014 i.e. for six year period have been used taken viz. IBA bulletin, statistics published by Reserve Bank of India, annual reports published by the banks.

\subsection{Input Attribute values}

The four Input attributes are Debt-Equity Ratio, Net NPA to Net Advances, Net NPA to Total Assets, and Total Investments to Total Assets, and the values of these attributes are presented in following table.

Table 1

\begin{tabular}{|l|c|c|c|c|}
\hline \multicolumn{1}{|c|}{ Bank name } & $\begin{array}{c}\text { Debt-Equity } \\
\text { ratio }\end{array}$ & $\begin{array}{c}\text { Net NPA to } \\
\text { Net Advance }\end{array}$ & $\begin{array}{c}\text { Net NPA to } \\
\text { Total assets }\end{array}$ & $\begin{array}{c}\text { Total Inv. } \\
\text { to Total } \\
\text { Assets }\end{array}$ \\
\hline Allahabad Bank & 0.86 & 1.75 & 1.09 & 29.63 \\
\hline Andhra bank & 1.23 & 1.20 & 0.79 & 24.44 \\
\hline Bank of Baroda & 0.94 & 0.72 & 0.45 & 20.56 \\
\hline Bank of India & 1.48 & 1.37 & 0.86 & 22.59 \\
\hline Bank of Maharashtra & 1.25 & 1.19 & 0.73 & 28.89 \\
\hline Canara Bank & 0.85 & 1.57 & 0.91 & 26.66 \\
\hline Central Bank of India & 1.01 & 2.05 & 1.28 & 27.58 \\
\hline Corporation Bank & 1.52 & 0.90 & 0.55 & 29.80 \\
\hline Dena Bank & 0.81 & 1.37 & 0.85 & 27.57 \\
\hline IDBI Bank & 3.00 & 1.45 & 0.88 & 29.70 \\
\hline Indian Bank & 0.26 & 1.13 & 0.73 & 26.86 \\
\hline Indian Overseas bank & 1.69 & 2.02 & 1.28 & 26.27 \\
\hline Oriental bank of commerce & 0.53 & 1.63 & 1.02 & 28.08 \\
\hline Punjab \& Sind bank & 0.96 & 1.33 & 0.82 & 29.12 \\
\hline Punjab national bank & 1.22 & 1.37 & 0.88 & 26.18 \\
\hline State bank of Bikaner \& Jaipur & 1.11 & 1.53 & 1.06 & 22.71 \\
\hline
\end{tabular}




\begin{tabular}{|l|c|c|c|c|}
\hline \multicolumn{1}{|c|}{ Bank name } & $\begin{array}{c}\text { Debt-Equity } \\
\text { ratio }\end{array}$ & $\begin{array}{c}\text { Net NPA to } \\
\text { Net Advance }\end{array}$ & $\begin{array}{c}\text { Net NPA to } \\
\text { Total assets }\end{array}$ & $\begin{array}{c}\text { Total Inv. } \\
\text { to Total } \\
\text { Assets }\end{array}$ \\
\cline { 1 - 4 } State bank of Hyderabad & 1.15 & 1.35 & 0.85 & 25.84 \\
\hline State bank of India & 1.61 & 1.94 & 1.22 & 24.65 \\
\hline State bank of Mysore & 1.10 & 1.80 & 1.19 & 25.59 \\
\hline State bank of Patiala & 1.41 & 1.49 & 0.97 & 22.59 \\
\hline State bank of Travancore & 1.57 & 1.37 & 0.90 & 26.40 \\
\hline Syndicate Bank & 1.46 & 1.02 & 0.68 & 22.53 \\
\hline Uco Bank & 1.28 & 1.95 & 1.22 & 27.36 \\
\hline Union bank of India & 1.15 & 1.33 & 0.86 & 25.92 \\
\hline United bank of India & 0.66 & 2.73 & 1.53 & 30.91 \\
\hline Vijaya Bank & 0.79 & 1.38 & 0.83 & 29.63 \\
\hline
\end{tabular}

\subsection{Output Attributes}

Capital Adequacy ratio, Advances to Assets, Govt. securities to Total Investment, Business per Employee, Profit per Employee, Credit Deposit Ratio, Return on net worth, Return on assets, NIM to Total Assets, Operating profit to total assets, Interest income to Total income, Liquid Assets to Total Assets, Govt. securities to total assets, Liquid Assets to Total deposits, Liquid Assets to Demand Deposits are considered as output attributes and are presented in the following table 2. 


\begin{tabular}{|c|c|c|c|c|c|c|c|c|c|c|c|c|c|c|c|}
\hline 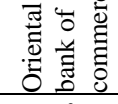 & $\begin{array}{l}: \\
\stackrel{0}{0} \\
\text { ] }\end{array}$ & a] & $\begin{array}{l}1 \\
0 \\
\vdots \\
\vdots\end{array}$ & $\begin{array}{l}\stackrel{f}{+} \\
\dot{J}\end{array}$ & 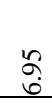 & $\begin{array}{l} \pm \\
\infty \\
\stackrel{2}{2}\end{array}$ & $\begin{array}{l}\circ \\
\stackrel{0}{0} \\
\end{array}$ & $\begin{array}{l}\hat{\imath} \\
\hat{0}\end{array}$ & $\overrightarrow{\vec{i}}$ & $\stackrel{\infty}{\infty}$ & $\begin{array}{l}\stackrel{2}{a} \\
\dot{a}\end{array}$ & $\stackrel{n}{2}$ & $\begin{array}{l}0 \\
\tilde{i} \\
\text { in }\end{array}$ & $=\vec{a}$ & 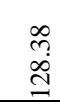 \\
\hline 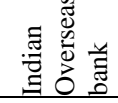 & $\begin{array}{l}\overrightarrow{7} \\
\dot{m}\end{array}$ & $\begin{array}{l}2 \\
6 \\
6\end{array}$ & $\begin{array}{l}f \\
f \\
\dot{0}\end{array}$ & $\begin{array}{l}\stackrel{9}{0} \\
\stackrel{9}{9}\end{array}$ & m. & $\begin{array}{l}\vec{F} \\
\stackrel{0}{0}\end{array}$ & 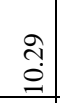 & $\begin{array}{l}\tilde{n} \\
0 \\
0\end{array}$ & $\stackrel{\circ}{i}$ & ه్ర & $\begin{array}{l}\dot{b} \\
\dot{a}\end{array}$ & $\begin{array}{l}0 \\
0 \\
0 \\
0\end{array}$ & $\begin{array}{l}\vec{b} \\
\vec{i}\end{array}$ & $\begin{array}{l}\bar{m} \\
\infty\end{array}$ & $\begin{array}{l}\text { ț } \\
\stackrel{+}{0}\end{array}$ \\
\hline 䔅辟 & $\begin{array}{l}\infty \\
\stackrel{2}{2} \\
\end{array}$ & 告 & $\begin{array}{l}\overrightarrow{0} \\
\dot{0}\end{array}$ & @ి & $\stackrel{\infty}{\stackrel{\sim}{\sim}}$ & $\begin{array}{l}\mathscr{m} \\
0 \\
i \\
\tilde{i}\end{array}$ & $\begin{array}{c}0 \\
\substack{n \\
n}\end{array}$ & $\stackrel{?}{m}$ & $\overrightarrow{\widehat{a}}$ & $\tilde{\widetilde{N}}$ & $\begin{array}{l}\tilde{n} \\
\vdots \\
\infty\end{array}$ & 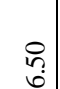 & $\begin{array}{l}\vec{t} \\
\stackrel{d}{d}\end{array}$ & in & $\stackrel{8}{\stackrel{\Xi}{\Xi}}$ \\
\hline $\begin{array}{l}\overrightarrow{0} \\
\hat{E}\end{array}$ & 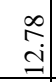 & 형 & ?ై & $\underset{\vec{d}}{\stackrel{J}{d}}$ & nุ & 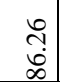 & $=$ & $\tilde{0}$ & $\underset{I}{\stackrel{I}{I}}$ & $\stackrel{q}{+}$ & 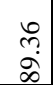 & $\hat{a}$ & $\begin{array}{c}\mathbf{b} \\
\mathrm{d} \\
\mathbf{d}\end{array}$ & $\begin{array}{l}q \\
\dot{\sigma} \\
\infty\end{array}$ & $\begin{array}{l}\stackrel{\infty}{\infty} \\
\stackrel{i}{i}\end{array}$ \\
\hline 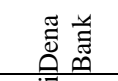 & 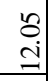 & 年 & 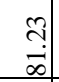 & $\stackrel{\vec{m}}{=}$ & $\stackrel{\infty}{\stackrel{\infty}{i n}}$ & $\begin{array}{l}8 \\
8 \\
8\end{array}$ & $\begin{array}{l}\sigma \\
\sigma \\
\sigma\end{array}$ & $\bar{\sigma}$ & तె & $\stackrel{\leftrightarrow}{n}$ & $\begin{array}{l}0 \\
\stackrel{0}{2} \\
2\end{array}$ & $\stackrel{\vartheta}{i}$ & $\begin{array}{l}\hat{\tilde{n}} \\
\tilde{\mathrm{d}}\end{array}$ & $\left.\begin{array}{l}\bar{\infty} \\
\dot{\infty}\end{array}\right]$ & $\begin{array}{l}\bar{n} \\
\stackrel{0}{0}\end{array}$ \\
\hline 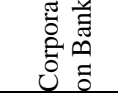 & ले. & : & 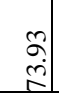 & $\begin{array}{l}0 \\
i \\
i\end{array}$ & $\begin{array}{l}: \\
: \\
\infty\end{array}$ & $\begin{array}{l}\vec{i} \\
\dot{2}\end{array}$ & $\begin{array}{l}\tilde{n} \\
\underline{\bullet} \\
\underline{-1}\end{array}$ & ڤ్రి & $\stackrel{\infty}{-\infty}$ & $\stackrel{t}{\stackrel{f}{*}}$ & $\begin{array}{l}\infty \\
\substack{\infty \\
\infty \\
\infty}\end{array}$ & $\stackrel{\leftrightarrow}{\infty}$ & $\begin{array}{l}8 \\
\text { i } \\
\text { d }\end{array}$ & के & $\underset{\infty}{n}$ \\
\hline 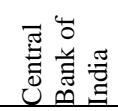 & $\stackrel{-}{a}$ & $\begin{array}{l}7 \\
0 \\
0\end{array}$ & $\begin{array}{l}n \\
\\
0 \\
\infty\end{array}$ & 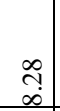 & $\stackrel{R}{i}$ & $\begin{array}{l}\text { ب̃ } \\
\stackrel{1}{r} \\
\end{array}$ & $\begin{array}{l}3 \\
0 \\
\infty\end{array}$ & ț & $\underset{\sim}{\infty}$ & $\stackrel{ \pm}{ت}$ & $\begin{array}{l}n \\
n \\
a \\
\vdots\end{array}$ & $\begin{array}{l}0 \\
0 \\
0 \\
0\end{array}$ & $\begin{array}{l}\infty \\
\stackrel{+}{\dot{j}} \\
\dot{d}\end{array}$ & $\tilde{n}$ & $\begin{array}{l}\tilde{0} \\
\stackrel{8}{0}\end{array}$ \\
\hline 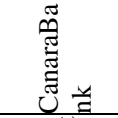 & 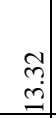 & : & $\begin{array}{l}0 \\
0 \\
\dot{\infty} \\
\end{array}$ & $\stackrel{a}{=}$ & $\underset{r}{\stackrel{t}{r}}$ & $\underset{\stackrel{f}{+}}{\stackrel{f}{r}}$ & $\begin{array}{c}n \\
\tilde{n} \\
\underline{6}\end{array}$ & $\stackrel{\vec{\sigma}}{-}$ & $\underset{\substack{\infty \\
-\infty}}{ }$ & مْ & $\begin{array}{l}\hat{b} \\
\dot{0}\end{array}$ & $\begin{array}{l}8 \\
0 \\
\infty\end{array}$ & $\begin{array}{c}m \\
\dot{i} \\
\end{array}$ & तू & $\begin{array}{l}\infty \\
\infty \\
\infty \\
\stackrel{\Xi}{\simeq} \\
\end{array}$ \\
\hline 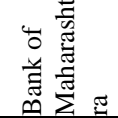 & $\begin{array}{l}\text { J } \\
\text { in }\end{array}$ & 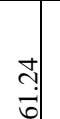 & 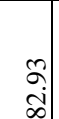 & $\begin{array}{l}\infty \\
\infty \\
\infty\end{array}$ & ते & $\stackrel{f}{\stackrel{f}{*}}$ & $\stackrel{\infty}{\underset{\mathrm{I}}{\mathrm{I}}}$ & $\begin{array}{l}\infty \\
n \\
? \\
0\end{array}$ & $\hat{\tilde{m}}$ & $\stackrel{?}{\stackrel{f}{+}}$ & $\begin{array}{l}8 \\
\\
\end{array}$ & 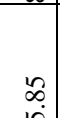 & $\begin{array}{c}\hat{\sigma} \\
\vec{j}\end{array}$ & $\stackrel{\infty}{\stackrel{6}{6}}$ & $\underset{⿱ t}{\stackrel{5}{5}}$ \\
\hline 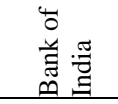 & $\begin{array}{l}n \\
\text { in } \\
\end{array}$ & ज: & \begin{tabular}{l}
7 \\
\multirow{2}{*}{} \\
$\dot{\infty}$
\end{tabular} & $\stackrel{\vec{\omega}}{\vec{m}}$ & त్రి & 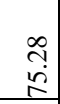 & $\begin{array}{c}\stackrel{2}{2} \\
\dot{g}\end{array}$ & $\begin{array}{l}\widetilde{1} \\
0 \\
0 \\
0\end{array}$ & $\stackrel{丶}{\circ}$ & $\stackrel{n}{g}$ & $\begin{array}{l}n \\
n \\
\infty \\
\infty\end{array}$ & $\begin{array}{l}\stackrel{\infty}{\sim} \\
\stackrel{1}{\varrho}\end{array}$ & $\begin{array}{l}\infty \\
\stackrel{1}{\beth} \\
\stackrel{2}{二}\end{array}$ & $\begin{array}{l}\text { İ } \\
\text { İ }\end{array}$ & $\underset{\stackrel{N}{N}}{\stackrel{N}{N}}$ \\
\hline 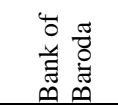 & $\begin{array}{l}\overline{\grave{g}} \\
\dot{g}\end{array}$ & $\begin{array}{l}7 \\
\text { त् }\end{array}$ & $\begin{array}{l}\hat{\partial} \\
\grave{j}\end{array}$ & $\begin{array}{l}\infty \\
\stackrel{\infty}{2} \\
\end{array}$ & $\bar{a}$ & $\begin{array}{l}\hat{b} \\
\text { in } \\
\end{array}$ & 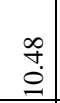 & $\stackrel{g}{a}$ & $\begin{array}{l}8 \\
i\end{array}$ & $\stackrel{尺}{I}$ & $\begin{array}{c}\vec{J} \\
\dot{\infty} \\
\infty\end{array}$ & $\begin{array}{l}\overleftarrow{6} \\
\dot{ \pm}\end{array}$ & 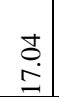 & $\begin{array}{c}5 \\
\stackrel{\Xi}{\Xi}\end{array}$ & 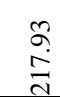 \\
\hline 焉 & $\overline{\mathrm{d}}$ & 월 & t. & 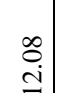 & $\underset{\infty}{\sigma}$ & 志 & $\bar{a}$ & $\stackrel{s}{o}$ & $i n$ & $\stackrel{\infty}{\circ}$ & 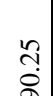 & $\stackrel{?}{q}$ & $\underset{\vec{i}}{\vec{i}}$ & $\hat{0}$ & $\begin{array}{l}n \\
\text { àj } \\
\text { İ }\end{array}$ \\
\hline 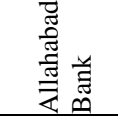 & 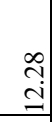 & 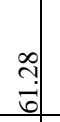 & $\begin{array}{l}\stackrel{a}{\alpha} \\
\stackrel{\rho}{\rho}\end{array}$ & $\begin{array}{l}\hat{\varrho} \\
\varrho\end{array}$ & 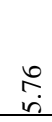 & $\begin{array}{l}\tilde{m} \\
\stackrel{2}{r}\end{array}$ & $\stackrel{\vartheta}{\overrightarrow{ \pm}}$ & $\begin{array}{l}8 \\
\vdots \\
0\end{array}$ & 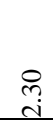 & $\stackrel{t}{g}$ & $\begin{array}{l}n \\
\dot{0} \\
\infty\end{array}$ & $\hat{\imath}$ & $\begin{array}{c}\hat{j} \\
\tilde{n}\end{array}$ & $\stackrel{尺}{i}$ & 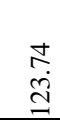 \\
\hline 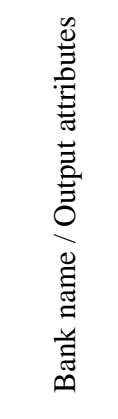 & 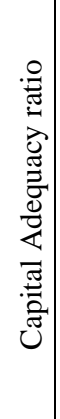 & 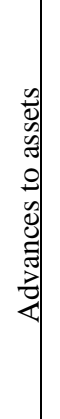 & 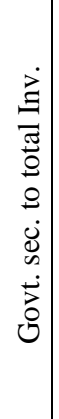 & 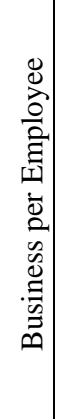 & 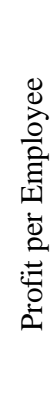 & 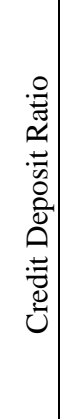 & 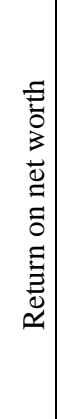 & 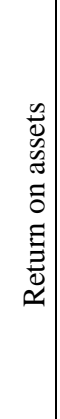 & 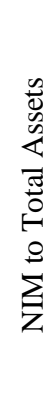 & 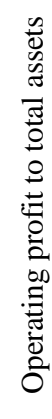 & 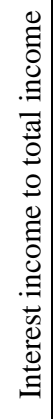 & 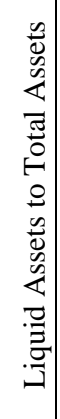 & 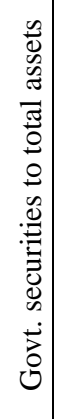 & 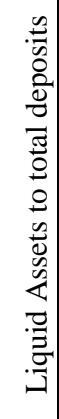 & $\begin{array}{l}0 \\
0 \\
0 \\
0 \\
0 \\
0 \\
0 \\
0 \\
0 \\
0 \\
0 \\
0 \\
0 \\
0 \\
0 \\
0 \\
0\end{array}$ \\
\hline
\end{tabular}


Performance Evaluation of Banks Through Four Phased DEA - A Case Study

\begin{tabular}{|c|c|c|c|c|c|c|c|c|c|c|c|c|c|c|c|}
\hline 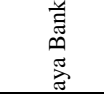 & $\begin{array}{l}\stackrel{9}{\mathrm{i}} \\
\mathrm{d}\end{array}$ & $\begin{array}{l}\hat{b} \\
0 \\
0\end{array}$ & $\begin{array}{l}1 \\
0 \\
\dot{2}\end{array}$ & $\begin{array}{l}\stackrel{f}{0} \\
\stackrel{0}{0}\end{array}$ & 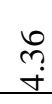 & $\begin{array}{l}\hat{b} \\
\hat{\vdots} \\
\end{array}$ & $\begin{array}{l}\infty \\
0 \\
0\end{array}$ & $\begin{array}{l}\overline{0} \\
0\end{array}$ & $\begin{array}{l}\infty \\
\infty \\
-\end{array}$ & 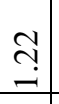 & $\begin{array}{l}\vec{b} \\
\vec{a}\end{array}$ & $\underset{\sim}{\stackrel{f}{\tau}}$ & $\begin{array}{l}\hat{\infty} \\
\tilde{\Sigma} \\
\end{array}$ & $\begin{array}{l}0 \\
+ \\
\infty \\
\end{array}$ & \\
\hline 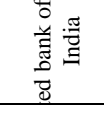 & 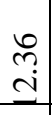 & $\begin{array}{l}n \\
\vdots \\
i \\
0\end{array}$ & 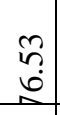 & $\begin{array}{l}0 \\
\infty \\
\infty \\
\infty\end{array}$ & $\stackrel{\sigma}{\sigma}$ & $\begin{array}{l}b \\
b \\
\dot{b}\end{array}$ & $\stackrel{n}{r}$ & $\begin{array}{l}0 \\
\stackrel{1}{0} \\
\end{array}$ & $\begin{array}{l}\tilde{r} \\
\ddot{i}\end{array}$ & กิ & 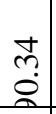 & $\underset{\infty}{=}$ & $\begin{array}{l}8 \\
b \\
\end{array}$ & $\begin{array}{l}\tilde{a} \\
a\end{array}$ & \\
\hline 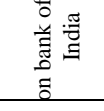 & 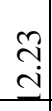 & $\begin{array}{l}\hat{0} \\
\dot{t}\end{array}$ & $\begin{array}{l}2 \\
\infty \\
\infty \\
\infty\end{array}$ & 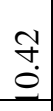 & రి & $\begin{array}{l}8 \\
\dot{1} \\
i\end{array}$ & $\begin{array}{l}\circ \\
\stackrel{+}{+}\end{array}$ & $\stackrel{n}{0}$ & $\begin{array}{l} \pm \\
i \\
\end{array}$ & $\underset{-\infty}{-\infty}$ & $\begin{array}{l}f \\
\stackrel{2}{a} \\
2\end{array}$ & $\underset{ָ}{\stackrel{N}{r}}$ & 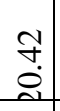 & $\begin{array}{l}\mathscr{f} \\
\infty \\
\infty\end{array}$ & \\
\hline 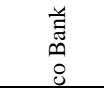 & $\begin{array}{l}\hat{0} \\
\dot{d}\end{array}$ & 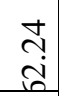 & $\begin{array}{l}r \\
i \\
i\end{array}$ & $\begin{array}{l}0 \\
0 \\
0\end{array}$ & $\stackrel{\overbrace{}}{\sim}$ & $\tilde{n}$ & $\stackrel{n}{i}$ & $\begin{array}{l}\dot{t} \\
\dot{0}\end{array}$ & $\stackrel{2}{2}$ & $\stackrel{\leftrightarrow}{n}$ & $\begin{array}{l}\stackrel{a}{ } \\
i \\
i\end{array}$ & $\stackrel{n}{n}$ & $\begin{array}{c}\mathfrak{y} \\
\mathrm{i} \\
\end{array}$ & $\begin{array}{l}8 \\
\dot{\infty} \\
\infty\end{array}$ & \\
\hline 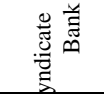 & $\begin{array}{l}\mathfrak{g} \\
i \\
i\end{array}$ & $\begin{array}{l}\hat{\infty} \\
0 \\
0\end{array}$ & $\begin{array}{c}t \\
\dot{b} \\
\tilde{n}\end{array}$ & $\begin{array}{l}\hat{\imath} \\
0 \\
0\end{array}$ & $\stackrel{7}{n}$ & $\begin{array}{c}a \\
\mathbf{1} \\
\mathbf{1}\end{array}$ & $\stackrel{+}{\stackrel{+}{ \pm}}$ & $\begin{array}{l}\bar{\infty} \\
0 \\
\end{array}$ & $\begin{array}{l}\text { त̂ } \\
\text {. }\end{array}$ & $\stackrel{n}{n}$ & $\begin{array}{l}\infty \\
\\
\\
\end{array}$ & $\begin{array}{l}\overrightarrow{0} \\
\infty \\
\end{array}$ & $\begin{array}{l}2 \\
\vdots \\
0\end{array}$ & సे & \\
\hline 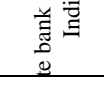 & $\stackrel{\infty}{\dot{m}}$ & $\begin{array}{l}\infty \\
\infty \\
i \\
i\end{array}$ & $\begin{array}{l}\bar{a} \\
\dot{2}\end{array}$ & $\begin{array}{c}m \\
\infty \\
\end{array}$ & $\underset{+}{+}$ & $\stackrel{n}{n}$ & $\begin{array}{l}\hat{b} \\
i \\
i\end{array}$ & $\begin{array}{l}n \\
\infty \\
0\end{array}$ & तે & $\hat{a}$ & $\begin{array}{l}\infty \\
0 \\
\dot{\infty}\end{array}$ & $\underset{\infty}{\stackrel{\Upsilon}{\infty}}$ & $\begin{array}{l}+ \\
\vdots \\
\vdots \\
\end{array}$ & $\begin{array}{l}n \\
\tilde{n} \\
0\end{array}$ & \\
\hline 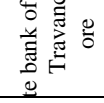 & $\begin{array}{r}\mathfrak{i} \\
i\end{array}$ & $\begin{array}{l}o \\
\dot{b} \\
n\end{array}$ & $\begin{array}{l}\infty \\
0 \\
\dot{+}\end{array}$ & $\begin{array}{c}\hat{N} \\
\infty\end{array}$ & $\begin{array}{l}\text { ñ } \\
\text { in }\end{array}$ & $\bar{a}$ & $\underset{5}{0}$ & $\begin{array}{l}\infty \\
\infty \\
0 \\
0\end{array}$ & $\hat{n}$ & $\begin{array}{l}\bar{\sigma} \\
\end{array}$ & $\begin{array}{l}? \\
\stackrel{8}{8}\end{array}$ & $\begin{array}{l}0 \\
+ \\
i n \\
\end{array}$ & 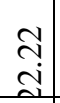 & ?్ֶ & \\
\hline 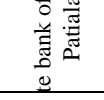 & 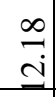 & $\begin{array}{l}\vec{v} \\
\dot{t}\end{array}$ & $\stackrel{2}{2}$ & $\begin{array}{l}\exists \\
0\end{array}$ & $\begin{array}{l}\tilde{\sigma} \\
\dot{+}\end{array}$ & $\begin{array}{l}\hat{\infty} \\
\stackrel{5}{5}\end{array}$ & $\begin{array}{l}\text { mे } \\
\end{array}$ & $\begin{array}{l}\stackrel{0}{0} \\
\stackrel{0}{0}\end{array}$ & $\begin{array}{l}\stackrel{+}{n} \\
\text { in }\end{array}$ & $\begin{array}{l}\mathbb{J} \\
\stackrel{-}{*}\end{array}$ & $\begin{array}{l}\infty \\
\grave{2} \\
\grave{\Omega}\end{array}$ & $\begin{array}{l}\vec{b} \\
\dot{b}\end{array}$ & 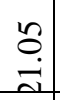 & $\begin{array}{l} \pm \\
\infty \\
\infty\end{array}$ & \\
\hline 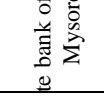 & $\begin{array}{l}n \\
\stackrel{n}{d} \\
\end{array}$ & $\begin{array}{l}0 \\
n \\
n \\
n\end{array}$ & $\begin{array}{l}\infty \\
n \\
n \\
n\end{array}$ & 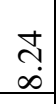 & $\begin{array}{c}\infty \\
\infty \\
\end{array}$ & $\begin{array}{l}n \\
n \\
\infty \\
n\end{array}$ & $\hat{o}$ & $\stackrel{2}{0}$ & $\begin{array}{l}\infty \\
\infty \\
i\end{array}$ & $\begin{array}{l}0 \\
\infty \\
-\end{array}$ & $\begin{array}{l}5 \\
\stackrel{0}{8}\end{array}$ & $\underset{+}{\stackrel{\sigma}{+}}$ & $\begin{array}{c}\vec{J} \\
\dot{d}\end{array}$ & $\begin{array}{l}\hat{a} \\
\dot{n}\end{array}$ & \\
\hline 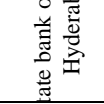 & $\frac{m}{m}$ & $\begin{array}{l}\stackrel{R}{i} \\
i\end{array}$ & $\begin{array}{l}\vec{a} \\
\tilde{m} \\
\infty \\
\infty\end{array}$ & $\begin{array}{l}\hat{\sigma} \\
0 \\
0\end{array}$ & రి & $\stackrel{m}{2}$ & $\stackrel{\infty}{\stackrel{\infty}{a}}$ & $\begin{array}{l}\infty \\
0 \\
0\end{array}$ & $\bar{a}$ & $\begin{array}{l}8 \\
\text { i } \\
\end{array}$ & $\begin{array}{l}\stackrel{9}{+} \\
\stackrel{2}{2}\end{array}$ & $\stackrel{⿱ 亠 乂}{\sim}$ & $\begin{array}{l}\stackrel{2}{\vec{i}} \\
\vec{i}\end{array}$ & $\underset{\infty}{t}$ & \\
\hline 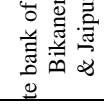 & $\begin{array}{l}+ \\
\infty \\
i \\
\end{array}$ & $\begin{array}{l}R \\
8 \\
0\end{array}$ & $\begin{array}{l}\infty \\
\stackrel{+}{ \pm} \\
+\end{array}$ & $\frac{n}{r}$ & $\frac{a}{n}$ & $\begin{array}{l}n \\
0 \\
\infty\end{array}$ & $\stackrel{0}{0}$ & $\stackrel{0}{0}$ & $\begin{array}{l}\stackrel{a}{ } \\
\text { in }\end{array}$ & $\stackrel{\infty}{\infty}$ & $\frac{a}{\dot{2}}$ & $\begin{array}{l}\infty \\
n \\
n\end{array}$ & $\vec{\nabla}$ & $\begin{array}{l}8 \\
0 \\
0\end{array}$ & \\
\hline 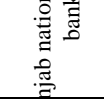 & $\begin{array}{l}\infty \\
\stackrel{\Xi}{\beth}\end{array}$ & $\begin{array}{l}8 \\
\dot{0} \\
\dot{0}\end{array}$ & $\begin{array}{c}\vec{T} \\
\stackrel{s}{\infty} \\
\infty\end{array}$ & $\begin{array}{l}\exists \\
0 \\
0\end{array}$ & กิ & $\begin{array}{l}\dot{b} \\
\dot{b} \\
i\end{array}$ & $\begin{array}{l}8 \\
\stackrel{0}{0}\end{array}$ & $\begin{array}{l}5 \\
-\end{array}$ & $\begin{array}{l}\tilde{\sigma} \\
i \\
i\end{array}$ & $\overrightarrow{\tilde{i}}$ & $\begin{array}{l}\tilde{b} \\
\infty \\
\infty \\
\infty\end{array}$ & $\underset{\sim}{\stackrel{d}{r}}$ & $\stackrel{2}{\vec{\lambda}}$ & $\begin{array}{l}0 \\
+ \\
\infty\end{array}$ & \\
\hline 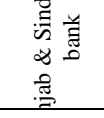 & $\begin{array}{c}\tilde{\sigma} \\
\stackrel{\leftrightarrow}{2}\end{array}$ & $\stackrel{\infty}{\longrightarrow}$ & 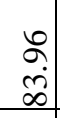 & $\stackrel{g}{\vec{g}}$ & $\begin{array}{l}\tilde{\sigma} \\
\dot{n}\end{array}$ & $\begin{array}{l}\infty \\
\stackrel{\infty}{2} \\
\stackrel{2}{2}\end{array}$ & $\begin{array}{l}\bar{\infty} \\
0 \\
0\end{array}$ & $\stackrel{\infty}{\stackrel{0}{0}}$ & 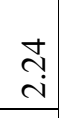 & $\vec{m}$ & $\begin{array}{l}\stackrel{n}{2} \\
\text { sid }\end{array}$ & $\bar{n}$ & 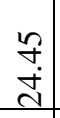 & 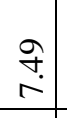 & \\
\hline 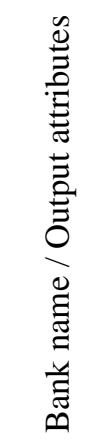 & 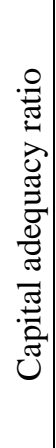 & 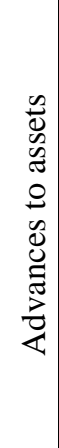 & $\begin{array}{c}\vec{\Xi} \\
\vec{\pi} \\
0 \\
0 \\
0 \\
\dot{0} \\
0 \\
\dot{0} \\
\dot{0} \\
\dot{0}\end{array}$ & 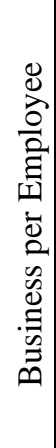 & 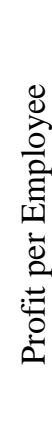 & 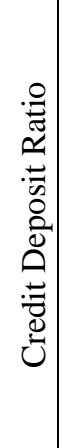 & $\begin{array}{l}\tilde{E} \\
0 \\
0 \\
0 \\
0 \\
0 \\
0 \\
0 \\
0 \\
0 \\
0\end{array}$ & 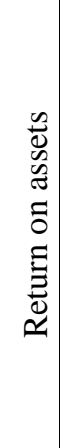 & $\begin{array}{l}n \\
0 \\
0 \\
0 \\
\Delta \\
0 \\
0 \\
0 \\
0 \\
0 \\
\vdots \\
z\end{array}$ & 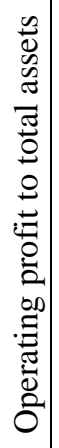 & 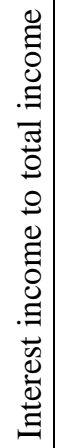 & 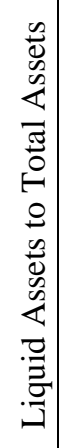 & 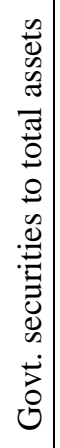 & 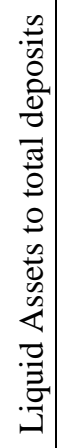 &. \\
\hline
\end{tabular}


Table 4 Input attribute values in four phase DEA approach

\begin{tabular}{|l|c|c|c|c|}
\hline \multicolumn{1}{|c|}{ Bank name } & $\begin{array}{c}\text { Debt-Equity } \\
\text { ratio }\end{array}$ & $\begin{array}{c}\text { Net NPA to } \\
\text { Net Advance }\end{array}$ & $\begin{array}{c}\text { Net NPA to } \\
\text { Total assets }\end{array}$ & $\begin{array}{c}\text { Total Inv. } \\
\text { to Total } \\
\text { Assets }\end{array}$ \\
\hline Allahabad Bank & 0.86 & 1.75 & 1.09 & 29.63 \\
\hline Andhra bank & 1.23 & 1.20 & 0.79 & 24.44 \\
\hline Bank of Baroda & 0.94 & 0.72 & 0.45 & 20.56 \\
\hline Bank of India & 1.48 & 1.37 & 0.86 & 22.59 \\
\hline Bank of Maharashtra & 1.25 & 1.19 & 0.73 & 28.89 \\
\hline Canara Bank & 0.85 & 1.57 & 0.91 & 26.66 \\
\hline Central Bank of India & 1.01 & 2.05 & 1.28 & 27.58 \\
\hline Corporation Bank & 1.52 & 0.90 & 0.55 & 29.80 \\
\hline Dena Bank & 0.81 & 1.37 & 0.85 & 27.57 \\
\hline IDBI Bank & 3.00 & 1.45 & 0.88 & 29.70 \\
\hline Indian Bank & 0.26 & 1.13 & 0.73 & 26.86 \\
\hline Indian Overseas bank & 1.69 & 2.02 & 1.28 & 26.27 \\
\hline $\begin{array}{l}\text { Oriental bank of } \\
\text { commerce }\end{array}$ & 0.53 & 1.63 & 1.02 & 28.08 \\
\hline Punjab \& Sind bank & 0.96 & 1.33 & 0.82 & 29.12 \\
\hline Punjab national bank & 1.22 & 1.37 & 0.88 & 26.18 \\
\hline $\begin{array}{l}\text { State bank of Bikaner } \\
\& \text { Jaipur }\end{array}$ & 1.11 & 1.53 & 1.06 & 22.71 \\
\hline $\begin{array}{l}\text { State bank of } \\
\text { Hyderabad }\end{array}$ & 1.15 & 1.35 & 0.85 & 25.84 \\
\hline State bank of India & 1.61 & 1.94 & 1.22 & 24.65 \\
\hline State bank of Mysore & 1.10 & 1.80 & 1.19 & 25.59 \\
\hline State bank of Patiala & 1.41 & 1.49 & 0.97 & 22.59 \\
\hline $\begin{array}{l}\text { State bank of } \\
\text { Travancore }\end{array}$ & 1.57 & 1.37 & 0.90 & 26.40 \\
\hline Syndicate Bank & 1.46 & 1.02 & 0.68 & 22.53 \\
\hline Uco Bank & 1.28 & 1.95 & 1.22 & 27.36 \\
\hline Union bank of India & 1.15 & 1.33 & 0.86 & 25.92 \\
\hline United bank of India & 0.66 & 2.73 & 1.53 & 30.91 \\
\hline Vijaya Bank & 0.79 & 1.38 & 0.83 & 29.63 \\
\hline
\end{tabular}


Table 5 Ranking order of banks in four phase DEA approach

\begin{tabular}{|c|c|c|c|c|c|c|c|}
\hline DMU & Bank name & $\begin{array}{c}\text { Ideal } \\
\text { Effici } \\
\text { ency } \\
\left(\theta_{1}^{*}\right)\end{array}$ & $\begin{array}{c}\text { Nadir } \\
\text { Effici } \\
\text { ency } \\
\left(\varphi_{n} *\right)\end{array}$ & $\begin{array}{c}\text { Best ideal } \\
\text { efficiency } \\
\left(\Theta_{p}^{*}\right)\end{array}$ & $\begin{array}{c}\text { Worst ideal } \\
\text { efficiency } \\
\left(\varphi_{p}^{*}\right)\end{array}$ & $\begin{array}{c}\text { Relative } \\
\text { Closeness } \\
\text { coefficien } \\
\mathbf{t} \\
\end{array}$ & $\underset{\mathbf{k}}{\operatorname{Ran}}$ \\
\hline DMU1 & Allahabad Bank & \multirow{26}{*}{2.95} & \multirow{26}{*}{0.1745} & 0.5543 & 0.1871 & 0.0052 & 22 \\
\hline DMU2 & Andhra bank & & & 0.6067 & 0.2329 & 0.0243 & 6 \\
\hline DMU3 & Bank of Baroda & & & 1 & 0.2629 & 0.0434 & 1 \\
\hline DMU4 & Bank of India & & & 0.5827 & 0.2464 & 0.0295 & 3 \\
\hline DMU5 & Bank of Maharashtra & & & 0.5041 & 0.187 & 0.0051 & 23 \\
\hline DMU6 & Canara Bank & & & 0.6778 & 0.212 & 0.0162 & 10 \\
\hline DMU7 & Central Bank of India & & & 0.3578 & 0.1951 & 0.0079 & 20 \\
\hline DMU8 & Corporation Bank & & & 0.7874 & 0.185 & 0.0048 & 24 \\
\hline DMU9 & Dena Bank & & & 0.6782 & 0.1973 & 0.0099 & 17 \\
\hline DMU10 & IDBI Bank & & & 0.575 & 0.1779 & 0.0014 & 25 \\
\hline DMU11 & Indian Bank & & & 1 & 0.2102 & 0.018 & 8 \\
\hline DMU12 & Indian Overseas bank & & & 0.3534 & 0.2163 & 0.0158 & 11 \\
\hline DMU13 & Oriental bank of commerce & & & 0.9095 & 0.2035 & 0.014 & 15 \\
\hline DMU14 & Punjab \& Sind bank & & & 0.6587 & 0.1969 & 0.0097 & 18 \\
\hline DMU15 & Punjab national bank & & & 0.4867 & 0.2135 & 0.0156 & 14 \\
\hline DMU16 & $\begin{array}{l}\text { State bank of Bikaner \& } \\
\text { Jaipur }\end{array}$ & & & 0.3667 & 0.2415 & 0.0253 & 5 \\
\hline DMU17 & State bank of Hyderabad & & & 0.5366 & 0.2206 & 0.0188 & 7 \\
\hline DMU18 & State bank of India & & & 0.2799 & 0.2192 & 0.0165 & 4 \\
\hline DMU19 & Ste bank of Mysore & & & 0.3582 & 0.2111 & 0.0139 & 12 \\
\hline DMU20 & State bank of Patiala & & & 0.4301 & 0.2434 & 0.0266 & 9 \\
\hline DMU21 & State bank of Travancore & & & 0.342 & 0.2161 & 0.0157 & 16 \\
\hline DMU22 & Syndicate Bank & & & 0.4908 & 0.2503 & 0.0299 & 2 \\
\hline DMU23 & UCO Bank & & & 0.4277 & 0.1975 & 0.009 & 19 \\
\hline DMU24 & Union bank of India & & & 0.5229 & 0.213 & 0.0156 & 13 \\
\hline DMU25 & United bank of India & & & 0.3907 & 0.1748 & 0.0001 & 26 \\
\hline DMU26 & Vijaya Bank & & & 0.642 & 0.1879 & 0.0058 & 21 \\
\hline
\end{tabular}

\section{RESULTS \& DISCUSSIONS}

The best relative efficiency of the ideal DMU $\left(\Theta 1^{*}\right)$ is determined by solving the optimization model as discussed in step 1 of methodology section for a set of 26 DMUs (Banks) with 4 inputs \& 15 outputs using LINGO solver. The best relative efficiency of the ideal DMU $\left(\Theta_{1}{ }^{*}\right)$, of 2.95 is obtained,

The worst relative efficiency of nadir DMU $\left(\varphi n^{*}\right)$ is determined by solving the optimization model as discussed in step 2 of methodology section for a set of 26 DMUs (Banks) with 4 inputs \& 15 outputs using LINGO solver. The worst relative efficiency of nadir DMU $\left(\varphi_{\mathrm{n}}{ }^{*}\right)$ of 0.1745 is obtained,

The best relative efficiencies of each $\operatorname{DMU}_{\mathrm{p}}\left(\Theta_{\mathrm{p}}{ }^{*}\right)$, is determined by solving the optimization model as discussed in step 3 of methodology section. And the worst relative efficiencies of each $\operatorname{DMU}_{\mathrm{p}}\left(\varphi_{\mathrm{p}}{ }^{*}\right)$, is determined by solving the optimization model as discussed in step 4 of methodology section. Relative closeness index of each 
Bank (DMU) is determined from the efficiencies obtained from the above models as discussed in step 5 of the methodology section. The results are shown in table 3 .

On the basis of these values, Bank 3(Bank of Baroda ) secured the first position with relative closeness coefficient 0.0433, followed by Bank 22- Syndicate Bank (0.0299), Bank of India (0.0293). United bank of India (0.000) secured the least position, followed by IDBI (0.0013), and Corporation Bank (0.0047).

\section{CONCLUSIONS}

In evaluating the function of the banks, many of the developed countries are now following uniform financial rating system along with other existing procedures and techniques. Banks are ranked according to the ratings obtained by them on the five factors. DEA is a methodology for measuring the relative efficiencies of a set of DMUs that use multiple inputs to produce multiple outputs. Due to its solid underlying theoretical basis and wide application to real world problems, DEA has been widely used to evaluate performance of banks. In this paper, the concept of the TOPSIS is integrated in to a four phase DEA framework to measure the efficiencies of a set of DMUs and rank them with input-output levels. The proposed framework is generic, structured and comprehensive and can be applied to analyze various DMU evaluation problems.

\section{REFERENCES}

[1] Adel Hatami-Marbini et al. (2010), "An ideal-seeking fuzzy data envelopment analysis framework", Elsevier Applied soft computing, Vol.10, pp.1062-1070.

[2] Arora, S.; and Kaur, S. (2006), "Financial Performance of Indian Banking Sector in Post-Reform Era”, The Indian Journal of Commerce, Vol.59 (1), pp.96-105.

[3] Bhattacharya and Chakra borty (2014), "A DEA-TOPSIS-based approach for performance evaluation of Indian Technical Institutes", Decision science letters, Vol. 3 (3), pp. 397-410.

[4] Chen et al. (2013), " the Analysis of Bank Business performance and market riskApplying Fuzzy dea”, Elsevier- Economic Modelling, Vol. 32, pp. 225-232.

[5] CA. Ruchi Gupta (2014); An Analysis of Indian Public Sector Banks Using Camel Approach; IOSR Journal of Business and Management (IOS-JBM); Vol. 16 (1.4), pp. 94-102.

[6] Iman Dabashi et al. (2013), “A Data Envelopment Analysis of Banks Performance in Iran", International Research Journal of Applied and Basic Sciences, Vol.4 (9), pp.2422-2426.

[7] K.V.N.Prasada, G. Ravinder (2012); A Camel Model Analysis of Nationalized Banks in India; International Journal of Trade and Commerce- iiartc; Vol.1 (1), pp. 23-33.

[8] Majid Karimzadeh (2012), "Efficiency Analysisby using dea model: Evidence from Indian Banks", Int. J. Latest trends Fin.Eco.Sc., Vol. 2 (3), pp. 228-237.

[9] Mishra Aswini Kumar, G. Sri Harsha, Shivi Anand and Neil Rajesh Dhruva (2012), "Analyzing Soundness in Indian Banking: A Camel Approach “, Research Journal of Management Sciences, Vol.1 (3), pp. 9-14. 
[10] Md. Anwarul kabir (2012), "Performance Analysis through camel Rating: A Comparative Study of Selected Private Commercial Banks in Bangladesh", Journal of Politics \& Governance, Vol.1 (2/3), pp. 16-25.

[11] Ritika et al. (2014), "Application of DEA for performance evaluation of Indian Micro finance institutions", Asian Journal of Management Research, Vol. 4 (3), 597-605.

[12] Priyanka and Dr D.P Jain. Empirical Study of Development Banks \& Entrepreneurship Promotion, International Journal of Industrial Engineering Research and Development, 6(1), 2015, pp. 01-08.

[13] Satyendra Sharma and Dr.Jayant Negi. Prioritization of Voice of Customers by Using Kano Questionnaire and Data Envelopment Analysis, International Journal of Industrial Engineering Research and Development, 4(1), 2013, pp. 01-09.

[14] Sanobar Anjum. A Study of Various Technique's For Measuring Banking Efficiency - A Significant Look At Data Envelopment Analysis, International Journal of Management, 3(2), 2012, pp. 01-09

[15] Sahila Chaudhry, Sultan Ultan singh, (2012), "Impact of reforms on the asset quality in Indian banking, International Journal of Multidisciplinary Research, Vol.2, pp. 13-31.Valerie Vierstraete (2012), "Efficiency in Human Development: a DEA", The European Journal of Comparative Economics, Vol. 9 (3), pp. 425443. 